\title{
El control de frecuencia es al menos equivalente al control de ritmo en fibrilación auricular persistente o recurrente
}

\begin{abstract}
Objetivo
Comparar la estrategia de control de frecuencia vs la de control de ritmo en pacientes con fibrilación auricular (FA), en cuanto al riesgo de muerte (objetivo primario) y secundariamente de accidente cerebrovascular (ACV).
\end{abstract}

\section{Fuentes de Datos}

MEDLINE, Registros Cochrane de Ensayos Controlados (CCTR) e International Pharmaceutical Abstracts (hasta 5/2003).

\section{Selección de estudios}

Dos investigadores independientes seleccionaron los estudios a incluir. Se excluyeron los estudios sobre FA postoperatoria y los que evaluaban procedimientos invasivos. Los desacuerdos se resolvieron por consenso o mediante un tercero. Se evaluaron criterios de calidad.

\section{Extracción de datos}

Un investigador extrajo los datos de los estudios y otro los verificaba. Los desacuerdos se resolvieron por consenso o mediante un tercero.

\section{Resultados principales}

Se incluyeron 5 ensayos clínicos controlados aleatorizados (ECCA) de 2850 estudios identificados, con 5239 pacientes de una edad media de 69 años con FA persistente o con alto riesgo de recurrencia seguidos por 1-3,5 años. La amiodarona fue el antiarrítmico más usado en la mayoría de los ECCA mientras que las drogas de control de frecuencia fueron más variadas. La proporción de pacientes asignados a control de ritmo mantenidos en ritmo sinusal al final del estudio varió sustancialmente (38 a 63,5\%). No se observaron diferencias estadísticamente significativas entre estrategias en cuanto a mortalidad pero sí una tendencia a favor del control de frecuencia vs control de ritmo (Tabla). Los resultaros no cambiaron significativamente al excluir al mayor estudio (AFFIRM), aunque sí la tendencia y una menor mortalidad (Frecuencia: 5,0\% vs Ritmo:4,5\%; OR 1,12 [0,64-1,94]. No hubo evidencia de heterogeneidad* entre ensayos.

Tabla. Resultados del meta-análisis control de frecuencia vs control de ritmo.

\begin{tabular}{l|c|c|c|c|c} 
Resultados & $\begin{array}{c}\text { Control de } \\
\text { Frecuencia } \\
\text { n/N (\%) }\end{array}$ & $\begin{array}{c}\text { Control de } \\
\text { Ritmo } \\
\text { n/N (\%) }\end{array}$ & OR & (IC 95\%) & p \\
\hline Muerte & $339 / 2609(13,0)$ & $339 / 2609(14,6)$ & 0,87 & $0,74-1,02$ & 0,09 \\
\hline ACV $^{*}$ & $78 / 2228(3,5)$ & $339 / 2609(3,9)$ & 0,50 & $0,14-1,83$ & 0,3 \\
\hline
\end{tabular}

OR: Odds Ratio. \#ACV: datos sólo disponibles de 3 estudios (AFFIRM, STAF y HOT CAFE)

\section{Conclusiones}

En pacientes con fibrilación auricular persistente, o con alto riesgo de recurrencia, la estrategia de control de frecuencia asociada a anticoagulación es al menos equivalente al control de ritmo con los antiarrítmicos disponibles para la prevención de resultados clínicos.

Fuente de financiamiento: no referida.

\section{Comentario}

Antes de abordar el comentario de ambos estudios debe mencionarse que las dos primeras línea terapéuticas aceptadas actualmente en FA son: 1) Cardioversión, mantenimiento del ritmo sinusal con antiarrítmicos apropiados (y generalmente anticoagulación); 2) Control de frecuencia y anticoagulación obligada. Uno de los estudios ( $\left.\mathrm{PIAF}^{1}\right)$, incluidos en el presente meta-análisis, no encontró diferencias sintomáticas ni de calidad de vida significativas al año de seguimiento pero sí una mayor tolerancia al ejercicio y más internaciones con amiodarona. Un $25 \%$ de suspensión y tan sólo $56 \%$ de mantenimiento de ritmo sinusal limitan sus conclusiones. Un estudio más grande (AFFIRM, $77,5 \%$ de la población metanalizada ${ }^{2}$, realizado en pacientes añosos, de alto riesgo de ACV concluyó que el control de ritmo no mejoró la sobrevida (de hecho existió una tendencia negativa). Por otro lado el control de frecuencia tuvo menos riesgo de efectos adversos por drogas, pero el mantenimiento del ritmo sinusal independientemente del uso de drogas antiarrítmicas se asoció a menor mortalidad ${ }^{3}$. Una evaluación económica basada en este estudio, mostró que el control de frecuencia era la estrategia dominante* pues además de una tendencia de beneficio ahorraba u\$s 5077 por persona (a 3,5 años de seguimiento ${ }^{4}$ ). Sin embargo las limitaciones de este estudio fueron varias, muchas en desmedro del control del ritmo. Si bien la tasa de mantenimiento de ritmo sinusal fue alta $(82 \%$ al año y $63 \%$ a los 5 años), la elección de la droga quedaba en manos de los médicos tratantes. La mayoría de los pacientes con las recomendaciones actuales debiera haber recibido amiodarona y aunque $2 / 3$ la recibieron en algún punto de los 5 años del estudio, sólo el 37,5\% la recibió inicialmente. La exigencia de anticoagulación era de sólo 4 semanas postcardioversión pero permanente en el grupo de control de frecuencia $(7.1 \%$ y $5,5 \%$ de ACV isquémicos respectivamente). Además la complejidad para el cumplimiento del protocolo en la rama control del ritmo explica el $20 \%$ global de cruces de estrategias en especial hacia control de frecuencia. Si hay un claro mensaje del análisis de la evidencia es que la mayoría de los ACV ocurren en pacientes sin anticoagulación o en rango subterapéutico y que independientemente de la estrategia seleccionada los pacientes con alto riesgo de ACV deben ser anticoagulados. Es infrecuente sostener ritmo permanentemente sinusal como para reducir el riesgo tromboembólico sin anticoagulación y además hasta un $25 \%$ de los ACV se producen en pacientes en ritmo sinusal. Otro estudio (RACE) evaluó una población diferente de pacientes añosos con FA persistente recurrente y también encontró una tendencia adversa del control del ritmo sinusal pero la amiodarona recién era utilizada si fracasaba en primera instancia con sotalol y en segunda con flecainida o propafenona, las cuales tienen un conocido efecto proarrítmico e incrementan la mortalidad en pacientes con cardiopatía ${ }^{5}$. Nuevamente las complicaciones tromboembólicas ocurrieron sólo en los pacientes de alto riesgo de ACV no anticoagulados (o en rango subterapéutico) independientemente de estar o no en ritmo sinusal y fueron mayores en el grupo control de ritmo que permitía la suspensión de la anticoagulación al mes de mantenimiento del ritmo sinusal. No hubo diferencias en la calidad de vida entre las dos estrategia en el AFFIRM ${ }^{6}$, el Europeo ${ }^{7}$ o el RACE ${ }^{8}$. El control del ritmo tampoco mejoraría la sobrevida, ni el riesgo de ACV, por lo que tampoco evitaría la anticoagulación, pero mejora la tolerancia al ejercicio, lo que podría ser importante en algunos pacientes con síntomas severos, cuando no son controlados con la otra estrategia. Por otro lado un $25 \%$ de los casos la FA puede mantenerse en ritmo sinusal por más de un año justificando brindarle una chance de cardioversión a la mayoría de los pacientes con un primer episodio y recurrencias sintomáticas de FA considerando los antiarrítmicos más seguros y eficaces en cada caso. Sin embargo debemos saber que el "costo" serán más internaciones y un tratamiento más complejo que el control de frecuencia. El segundo estudio comentado (SAFE-T), mostró que la amiodarona supera al sotalol manteniendo el ritmo sinusal. No halló diferencias estadísticamente significativas en cuanto a eventos adversos serios, y aunque no estuvo diseñado con ese fin y por ende de insuficiente poder, otros estudios tampoco hallaron diferencias y de hecho un meta-analisis (6500 pacientes post-infarto) mostró $13 \%$ de reducción de mortalidad con amiodarona $(p=0,03)^{9}$. También refuerza las evidencias de que manteniendo el ritmo sinusal mejora la capacidad de ejericio y contrasta con otras evidencias que no hallaron evidencias de beneficio en la calidad de 


\section{La amiodarona supera al sotalol manteniendo en ritmo sinusal a pacientes con fibrilación auricular}

\section{Objetivo}

Determinar si la amiodarona es superior al sotalol y si ambos son superiores al placebo manteniendo en ritmo sinusal a los pacientes con fibrilación auricular (FA) persistente.

\section{Diseño}

Ensayo clínico aleatorizado doble ciego, controlado con placebo; con un seguimiento de 1-4,5 años.

\section{Lugar \\ Estudio multicéntrico realizado en 20 centros ambulatorios de ve- teranos de EE.UU.}

\section{Pacientes}

Se asignaron 665 pacientes (99\% hombres y $89 \%$ blancos de $67 \pm 9$ años) anticoagulados por $F A=72$ hs a recibir amiodarona $(n=267)$, sotalol $(n=261)$, o placebo $(n=137)$. Se excluyeron pacientes con aleteo auricular, FA paroxística, insuficiencia cardíaca severa o con QT prolongado.

\section{Intervención}

Amiodarona: $800 \mathrm{mg} /$ día por 14 días, 600 los siguientes 14 días, 300 en el primer año y 200 mg/día de ahí en más. Sotalol: 80 mg 2 veces por día la primer semana y se duplicaba la dosis de allí en más. La medicación se suspendía y el paciente era seguido por un año si la FA recurría tras dos intentos de cardioversión.

\section{Medición de resultados principales}

Tiempo a la recurrencia de la FA desde el día 28 (cuando se practicaba una cardioversión eléctrica si aún no había revertido). Se evaluó calidad de vida mediante SF-36 y capacidad de ejercicio con ergometría.

\section{Resultados principales}

La cardioversión espontánea ocurrió en $27,1 \%$ con amiodarona, $24,2 \%$ con sotalol y $0,8 \%$ con placebo. La cardioversión eléctrica falló en $27,7 \%, 26,5 \%$, y $32,1 \%$, respectivamente. La adherencia fue $=95 \%$ pero significativamente mayor con amiodarona. La amiodarona fue superior al sotalol $(p<0,002)$ y al placebo $(p<0,001)$, y el sotalol superior al placebo $(p<0,001)$ (ver Tabla). El tiempo a la recurrencia de FA fue superior con amiodarona para todos los subgrupos excepto en los pacientes con cardiopatía isquémica en la que fue similar el sotalol. La restauración y mantenimiento del ritmo sinusal mejoraron significativamente ciertos aspectos de calidad de vida y la capacidad de ejercicio. No hubo diferencias significativas en los eventos adversos mayores entre los tres grupos.

Tabla 1. Tiempo a la recurrencia de FA en los pacientes cardiovertidos

\begin{tabular}{l|c|c|c}
$\begin{array}{c}\text { Resultados por "intención de } \\
\text { tratar"* (aunque "n"analizados < } \\
\text { aleatorizados) }\end{array}$ & $\begin{array}{c}\text { Amiodarona } \\
\text { (n=258) }\end{array}$ & $\begin{array}{c}\text { Sotalol } \\
(\mathbf{n}=\mathbf{2 4 4})\end{array}$ & $\begin{array}{c}\text { Placebo } \\
(\mathbf{n}=\mathbf{1 3 2})\end{array}$ \\
\hline Tiempo mediano a la recurrencia & 487 días & 74 días & 6 días \\
\hline Tasa de recurrencia al año & $48 \%$ & $68 \%$ & $87 \%$ \\
\hline NNT $^{\star}$ (IC 95\%) de recurrencia al & -vs Plac: $3(2-3)$ & $-v s$ Plac: $5(4-9)$ & - \\
a o(calculado por EVIDENCIA) & -vs Sot: $5(4-9)$ & - & - \\
\hline
\end{tabular}

\section{Conclusión}

La amiodarona y el sotalol son igualmente eficaces cardiovirtiendo la FA. La amiodarona es superior para mantener el ritmo sinusal pero tienen eficacia similar en pacientes con cardiopatía isquémica. El ritmo sinusal sostenido se asoció con una mejor calidad de vida y tolerancia al ejercicio.

Fuente de financiamiento: Centro Médico de Asuntos de Veteranos (Veterans Affairs Medical Center), y Laboratorios Berlex y Wyeth-Ayerst. vida (pero este análisis no fue por intención de tratar*). Mostró que la amiodarona y el sotalol favorecieron la cardioversión "espontánea" y eléctrica enfatizando el rol de los antiarrítmicos pre-cardioversión. Aunque sugerido por un análisis de subgrupo, pareciera que el sotalol podría tener una eficacia comparable a la amiodarona. Por último, el siempre sobrestimado perfil de eventos adversos de la amiodarona no difirió significativamente de los otros grupos como se ha visto al utilizar bajas dosis.

\section{Conclusión del comentador}

La nueva evidencia no elimina la estrategia de control del ritmo sinusal pero indica que no sería prudente la obstinación en la misma ante la falta de respuesta y acerca el punto de corte para elegir con más facilidad el control de frecuencia especialmente en los casos asintomáticos y recurrentes. El hallazgo de menor mortalidad en el subgrupo de los que logran mantenerse en ritmo sinusal estimula a la búsqueda de nuevas y más seguras estrate- gias para alcanzarlo. ${ }^{3}$

Los pacientes jóvenes con FA solitaria, FA altamente sintomática y quizás con IC severa (poco representados en los estudios descriptos) son aún potenciales candidatos a esta estrategia que conservaría las siguientes indicaciones ${ }^{10}$ :

1) primer episodio de FA (en ausencia de contraindicaciones), 2) pacientes sintomáticos a pesar de un adecuado control de frecuencia, 3) inadecuado control de frecuencia, 4) contraindicación de anticoagulación y 5) prevención del remodelamiento auricular en pacientes que se realizarán tratamientos no farmacológicos como el aislamiento de las venas pulmonares por radiofrecuencia, que parece promisorio en pacientes jóvenes sintomáticos si se demostrase una aceptable tasa de efectos adversos ${ }^{11}$. Se requerirán estudios adicionales en los que el control de ritmo incluya anticoagulación a la mayoría de los pacientes y amiodarona, que como se ha visto no es tan insegura como se le atribuye, para que podamos establecer claramente la primera línea de tratamiento.

Dr. Agustín Ciapponi [ Unidad de Medicina Familiar y Preventiva. Hospital Italiano de Buenos Aires . ]

* ver glosario

Ciapponi A. El control de frecuencia es al menos equivalente al control de ritmo en Fibrilación auricular persistente o recurrente. La amiodarona supera al sotalol mante-

niendo en ritmo sinusal a pacientes con fibrilación auricular. Evid actual pract ambul 2005;8:134-135. de Denus S, Sanoski CA, Carlsson J, Opolski G, Spinler SA. Rate vs Rhythm Control in Patients With Atrial Fibrillation: A Meta-analysis. Arch Intern Med. 2005;165:258-62. PMID: 15710787.Singh BN, Singh SN, Reda DJ, Tang XC, Lopez B, et al.; Sotalol Amiodarone Atrial Fibrillation Efficacy Trial (SAFE-T) Investigators.Amiodarone versus Sotalol for Atrial Fibrillation. N Engl J Med 2005;352(18):1861-72. PMID: 15872201.

\section{Referencias}

1. Hohnloser SH, Kuck KH. Atrial Fibrillation: Maintaining stability of sinus rhythm or ventricular rate control? The need for prospective data: the PIAF trial. Pace 1997;79:1989-1992).

2. Wyse DG, Waldo AL, DiMarco JP, Domanski MJ, Rosenberg Y, Schron EB, Kellen JC, Greene HL, Mickel MC, Dalquist JE, Corley SD.A comparison of rate control and rhythm control in patients with atrial fibrillation. N Engl J Med. 2002;347(23):1825-33.

3. Corley SD, Epstein AE, DiMarco JP, Domanski MJ, Geller N, Greene HL, et al. Relationships between sinus rhythm, treatment, and survival in the Atrial Fibrillation Follow-Up Investigation of Rhythm Management (AFFIRM) Study. Circulation. 2004;109:1509-13.

4. Marshall, DA, Levy AR, Vidaillet $\mathrm{H}$, et al. Cost-Effectiveness of Rhytm versus Rate Control in Atriall Fibrilation. Ann Intern Med 2004;143:653-661.

5. Van Gelder IC, Hagens VE, Bosker HA, et al. A comparison of rate control and rhythm control in patients with recurrent persistent atrial fibrillation. N Engl J Med 2002;347:1834-1840.

6. AFFIRM Investigators. Quality of life in atrial fibrillation. The AFFIRM Study. Am Heart J. 2004; [In press].

7. Gronefeld GC, Lilienthal J, Kuck KH, Hohnloser SH. Impact of rate versus rhythm control on quality of life in patients with persistent atrial fibrillation. Results from a prospective randomized study. Eur Heart J. 200324:14306. 8. Hagens VE, Ranchor AV, Van Sonderen E, Bosker HA, Kamp O, Tijssen JG, et al. Effect of rate or rhythm control on quality of life in persistent atrial fibrillation. Results from the Rate Control Versus Electrical Cardioversion (RACE) Study. J Am Coll Cardiol. 2004;43:241-7.

9. Amiodarone Trials Meta-Analysis Investigators. Effect of prophylactic amiodarone on mortality after acute myocardial infarction and in congestive heart failure: meta-analysis of individual data from 6500 patients in randomised trials. Lancet 1997:350:1417-1424.

10. Is There a Role for Maintaining Sinus Rhythm in Patients with Atrial Fibrillation? Zimetbaum P, Josephson, ME. Ann of Intern Med. 2004;141(9):720-726.

11. Wazni OM, Marrouche NF, Martin DO, Verma A, Bhargava M, et al. Radiofrequency ablation vs antiarrhythmic drugs as first-line treatment of symptomatic atrial fibrillation: a randomized trial. JAMA 2005;293(21):2634-40. 